\title{
Lower Magnetization Transfer Ratio in the Forceps Minor Is Associated with Poorer Gait Velocity in Older Adults
}

\author{
(D) S. Seiler, (D) Pirpamer, (D) B. Gesierich, (DE. Hofer, (D) M. Duering, (DD. Pinter, (DE. Jouvent, (D) F. Fazekas, (D).-F. Mangin, \\ (D) H. Chabriat, (DS. Ropele, and DR. Schmidt
}

\begin{abstract}
BACKGROUND AND PURPOSE: Gait disturbances in the elderly are disabling and a major public health issue but are poorly understood. In this multimodal MR imaging study, we used 2 voxel-based analysis methods to assess the voxelwise relationship of magnetization transfer ratio and white matter hyperintensity location with gait velocity in older adults.
\end{abstract}

MATERIALS AND METHODS: We assessed 230 community-dwelling participants of the Austrian Stroke Prevention Family Study. Every participant underwent 3T MR imaging, including magnetization transfer imaging. Voxel-based magnetization transfer ratio-symptom mapping correlated the white matter magnetization transfer ratio of each voxel with gait velocity. To assess a possible relationship between white matter hyperintensity location and gait velocity, we applied voxel-based lesion-symptom mapping.

RESULTS: We found a significant association between the magnetization transfer ratio within the forceps minor and gait velocity $(\beta=$ $0.134 ; 95 \% \mathrm{Cl}, 0.011-0.258 ; P=.033$ ), independent of demographics, general physical performance, vascular risk factors, and brain volume. White matter hyperintensities did not significantly change this association.

CONCLUSIONS: Our study provides new evidence for the importance of magnetization transfer ratio changes in gait disturbances at an older age, particularly in the forceps minor. The histopathologic basis of these findings is yet to be determined.

ABBREVIATIONS: $\mathrm{MNI}=$ Montreal Neurological Institute; $\mathrm{MTI}=$ magnetization transfer imaging; MTR $=$ magnetization transfer ratio; SPPB $=$ Short Physical Performance Battery; VLSM = voxel-based lesion symptom mapping; VMTRSM = voxel-based MTR symptom mapping; WMH = white matter hyperintensity

G ait abnormalities in older adults are common. ${ }^{1,2}$ They are associated with falls ${ }^{3,4}$ and represent a serious public health issue. ${ }^{1,5}$ A complex brain network manages supraspinal gait control. ${ }^{6}$ White matter hyperintensities (WMHs) are common and not necessarily related to clinical symptoms. However on a group

Received May 8, 2016; accepted after revision October 12.

From the Department of Neurology (S.S., L.P., E.H., D.P., F.F., S.R., R.S.) and Institute of Medical Informatics, Statistics and Documentation (E.H.), Medical University of Graz, Graz, Austria; Institute for Stroke and Dementia Research (B.G., M.D.), Klinikum der Universität München, Ludwig-Maximilians-University, Munich, Germany; Department of Neurology (E.J., H.C.), Institut National de la Santé et de la Recherche Médicale, UMR-740, Centre Hospitalo-Universitaire Lariboisière, Paris, France; and Neurospin (J.-F.M.), Commissariat à l'Energie Atomique et aux Energies Alternatives Saclay, Gif/Yvette, France.

This work was supported by the FP6 ERA-NET NEURON grant (01 EW1207/Mechanisms of Small Vessel Related Brain Damage and Cognitive Impairment) and by the Austrian Science Fund project 1904.

Parts of the data were presented at the Annual Meeting of the Austrian Alzheimer Society, October 24-25, 2014; Villach, Austria.

Please address correspondence to Reinhold Schmidt, MD, Department of Neurology, Clinical Division of Neurogeriatrics, Medical University Graz, Auenbruggerplatz 22, A-8036 Graz, Austria; e-mail: reinhold.schmidt@medunigraz.at

-- Indicates open access to non-subscribers at www.ajnr.org

三 Indicates article with supplemental on-line table.

http://dx.doi.org/10.3174/ajnr.A5036 level, widespread WMHs have been associated with gait dysfunction, probably as the consequence of disruption of the supraspinal gait network, ${ }^{7-9}$ and were related to gait performance in several studies, ${ }^{10-13}$ but results are conflicting. ${ }^{9,14,15}$ One explanation for conflicting results might be that as reported for cognitive decline, ${ }^{16-18}$ widespread, invisible, and highly variable microstructural changes in normal-appearing white matter also contribute to gait abnormalities in addition to visible lesions. This hypothesis is supported by 2 DTI studies that reported the higher mean diffusivity and lower fractional anisotropy in the genu of the corpus callosum to be correlated with poorer gait performance independent of visible WMHs. ${ }^{14,19}$

Complementary information on microstructural brain tissue alterations may come from magnetization transfer imaging (MTI). Other than DTI, which offers information on brain tissue organization, ${ }^{20}$ MTI offers information on tissue composition. ${ }^{21}$ Magnetization transfer ratio (MTR) is one of the few MR imaging measures that have been validated postmortem to represent a direct marker of myelin content. ${ }^{22}$

The only study on MTR and gait found that lower MTR was associated with poorer gait performance, independent of WMHs. $^{23}$ 
In this large multimodal MR imaging study, we used voxelbased MTR symptom mapping (VMTRSM) and voxel-based lesion symptom mapping (VLSM) to identify those brain areas in which MTR or WMH-related tissue alterations relate to gait velocity. We hypothesized that alterations, if any, would mainly be located within the frontal white matter because intact fontal subcortical pathways have been reported to be crucial for maintenance of gait performance at a higher level. ${ }^{6,24}$

\section{MATERIALS AND METHODS \\ Study Subjects}

The study sample is drawn from the Austrian Stroke Prevention Family Study, a prospective single-center community-based study designed to assess the cerebral effects of vascular risk factors in the healthy elderly population of the City of Graz, Austria. The Austrian Stroke Prevention Family Study represents an extension of the Austrian Stroke Prevention Study, which was established in 1991. ${ }^{25}$ Between 2006 and 2013, study participants of the Austrian Stroke Prevention Study and their first-degree relatives were invited to enter the Austrian Stroke Prevention Family Study. Individuals were excluded from the study if they had a history of neuropsychiatric disease, including previous cerebrovascular attacks and dementia, or abnormal neurologic examination findings, determined on the basis of a structured clinical interview and a physical and neurologic examination performed by a boardcertified neurologist. None of the study participants had a history or MR imaging findings suggestive of normal pressure hydrocephalus. There were also no subjects with a history or signs of heart failure in the study. None of the study participants had uncorrected visual impairment. A total of 381 individuals from 169 families were included in the study. The number of members per family ranged from 2 to 6 . All individuals underwent MR imaging, except for 26 who had contraindications. Thus, MTI scans were available in 355 subjects. The participants' ages ranged from 35 to 82 years. We focused on age-related decline in gait velocity and thus included all 230 subjects 60 years of age and older in the current analysis.

The ethics committee of the Medical University of Graz, Austria, approved the study protocol, and written informed consent was obtained from all subjects.

\section{Measurement of Gait Velocity}

Study participants were asked to walk a total distance of 8 meters with 3 turns at their usual, self-selected pace on level ground. None of the study participants needed walking aids. Time was measured with a stopwatch. The faster of the 2 trials was used for the subsequent analyses. We chose gait velocity because it can be measured quickly and in a clinical setting without instrumental efforts. It has been shown to be a good measure of mobility in elderly individuals. ${ }^{15}$

\section{Measurement of Cognition and General Physical Performance}

Scores of memory and executive function were assessed as described previously. ${ }^{26}$

General physical performance was assessed by using the Short Physical Performance Battery (SPPB). ${ }^{27}$

\section{Vascular Risk Factors}

Assessment of vascular risk factors included arterial hypertension, diabetes mellitus, hypercholesterolemia, hypertriglyceridemia, hyperuricemia, cardiac disease, peripheral vascular disease, and venous thrombotic disease and was determined on the basis of history and measurements at the examination as previously described. $^{26}$

\section{MR Imaging}

MR imaging was performed on a 3T whole-body scanner (Magnetom TrioTim; Siemens, Erlangen, Germany) and included conventional imaging and MTI. The MTI sequence was based on a spoiled $3 \mathrm{D}$ gradient-echo sequence $(\mathrm{TR}=40 \mathrm{~ms}$, $\mathrm{TE}=7.38 \mathrm{~ms}$, flip angle $=15^{\circ}$, number of sections $=40$, section thickness $=3 \mathrm{~mm}$, in-plane resolution $=0.86 \times 0.86$ $\mathrm{mm}$ ) performed with and without a Gaussian-shaped magnetization transfer saturation pulse.

The conventional protocol included an axial FLAIR sequence $(\mathrm{TR}=1000 \mathrm{~ms}, \mathrm{TE}=69 \mathrm{~ms}, \mathrm{TI}=2500 \mathrm{~ms}$, number of sections $=$ 40 , section thickness $=3 \mathrm{~mm}$, no intersection-gap, in-plane resolution $=0.86 \times 0.86 \mathrm{~mm}^{2}$ ) and a high-resolution T1-weighted $3 \mathrm{D}$ sequence with magnetization preparation and whole-brain coverage $(\mathrm{TR}=1900 \mathrm{~ms}, \mathrm{TE}=2.19 \mathrm{~ms}, \mathrm{TI}=900 \mathrm{~ms}$, flip angle $=$ $9^{\circ}$, isotropic resolution $=1 \mathrm{~mm}$ ).

For assessment of microbleeds, a $\mathrm{T}^{*}$ sequence was used $\left(\mathrm{TR}=35 \mathrm{~ms}, \mathrm{TE}=14.7 \mathrm{~ms}\right.$, flip angle $=15^{\circ}$, number of sections $=64$, section thickness $=2 \mathrm{~mm}$, no intersection-gap, inplane resolution $=0.90 \times 0.90 \mathrm{~mm}^{2}$ ).

\section{Visual MR Imaging Rating}

White matter hyperintensities and silent nonlacunar and lacunar infarcts were recorded on FLAIR images as previously described. ${ }^{26}$ Microbleeds were recorded on $\mathrm{T} 2{ }^{*}$-weighted images following the definition of Greenberg et al. ${ }^{28}$

\section{Generation of WMH and MTR Maps}

WMH maps were generated by using a custom-written Interactive Data Language program (DispImage; Exelis Visual Information Solutions, Boulder, Colorado) as described previously. ${ }^{29}$ Two highly experienced raters segmented WMHs on FLAIR images by combined region-growing and local thresholding following manual selection. ${ }^{29}$ The total lesion volume in cubic millimeters was calculated by multiplying the lesion area by the section thickness. WMH volumes in white matter tracts were calculated by overlaying the probabilistic white matter tract atlas (25\% probability), provided within the Oxford Centre for fMRI of the Brain Software Library (FSL; http://www.fmrib.ox.ac.uk/fsl), ${ }^{30}$ on the normalized WMH maps.

MTR maps were calculated according to the formula MTR = (M0 - MSS)/M0, where M0 represents the signal intensity of a voxel without any radiofrequency saturation and MSS is the signal intensity of the same voxel obtained with the radiofrequency saturation pulse. $^{21}$

For the subsequent steps, tools from FSL $^{31}$ were used.

Because we found that MTR provides good contrast in performing tissue segmentation, gray matter, white matter, and CSF partial volume maps were derived from the MTR-weighted scans 
by using FSL FAST (FMRIB Automated Segmentation Tool; http://fsl.fmrib.ox.ac.uk/fsl/fslwiki/FAST). ${ }^{32}$

The T1, FLAIR, and MTR-weighted scans were brainextracted by using the FSL Brain Extraction Tool (http://fsl.fmrib. ox.ac.uk/fsl/fslwiki/BET). ${ }^{33}$

The resulting skull-stripped T1-weighted images were nonlinearly registered to the Montreal Neurological Institute-152 standard space template (MNI 152) by using FSL FNIRT (FMRIB Nonlinear Image Registration Tool; http://fsl.fmrib.ox.ac.uk/fsl/ fslwiki/FNIRT). ${ }^{31}$

Then, the brain-extracted FLAIR and MTR scans were linearly registered to the corresponding brain-extracted T1-weighted images by using FLIRT (FMRIB Linear Image Registration Tool; http://www.fmrib.ox.ac.uk/). ${ }^{34}$

We used the transformation matrices from these steps to warp FLAIR and white matter MTR maps to the MNI-152 standard space template. WMH maps were transformed to the MNI-152 standard space template in the same way.

The resulting white matter MTR maps in standard space were eroded by 1 voxel to reduce partial volume effects resulting from "edge" voxels. To produce more normally distributed data, reduce noise, and account for the intersubject and registration variability, ${ }^{35}$ we smoothed the MTR maps with a 4-mm Gaussian kernel.

A mean white matter MTR mask was created and thresholded to exclude MTR values below $20 \% .{ }^{17}$ We chose this threshold to exclude voxels from CSF and to further reduce the spurious effects of partial volume effects caused by the white matter-gray matter transition zone.

Anatomic structures containing clusters of voxels in which MTR related significantly to gait velocity were localized by overlaying the probabilistic white matter tract atlas (25\% probability), provided within $\mathrm{FSL},{ }^{30}$ on the normalized white matter MTR maps. As in voxelwise analysis, we eroded the segmentations of these tracts by 1 voxel to reduce CSF artifacts at "edge" zones. The resulting "core" white matter mean MTR was used in subsequent analyses.

\section{Statistical Analysis}

General Statistical Analysis. Assumptions of normal distribution were tested with the Kolmogorov-Smirnov test. Normally distributed variables are reported as mean $\pm \mathrm{SD}$, and non-normally distributed variables, as median and interquartile range. $\mathrm{WMH}$ volume had a skewed distribution containing zero values; therefore, the value 2 was added to the volumes before natural logtransformation. To relate demographic, clinical, and imaging characteristics of the study participants to gait velocity, we categorized subjects into quartiles according to gait velocity distribution. One-way analysis of variance, with quartiles of walking speed as fixed factors and demographic, clinical, and imaging characteristics as outcome variables, and $\chi^{2}$ tests were performed to test significant associations with normally and non-normally distributed variables, respectively. Variables significantly $(P<$ $.05)$ associated with gait speed in these analyses were entered as covariates in the VMTRSM and ROI analyses described in the subsequent paragraphs. Correlations between MTR and gait velocity were calculated by using the Pearson correlation coefficient. Linear multiple regression analysis tested an independent rela- tionship between MTR and gait velocity. To test a possible doseeffect relationship, we used analysis of covariance with MTR quartiles as fixed factors and walking speed as the dependent variable. Age, sex, height, brain volume, general physical performance, presence of vascular risk factors, and MR imaging findings significantly associated with walking speed in the univariate analysis were entered as covariates in the regression analysis and the ANCOVA.

To assess mediating effects of executive function scores on the relationship between MTR and gait velocity, we used bootstrapped models as described by Preacher and Hayes. ${ }^{36}$

Voxel-Based MTR Symptom Mapping. To find associations between MTR values within a voxel and gait velocity, we used the permutation-based statistical interference tool for nonparametric testing (FSL Randomize tool; http://fsl.fmrib.ox.ac.uk/fsl/fslwiki/ Randomise/UserGuide). ${ }^{31}$ Five thousand permutations were performed to build the null distribution, and significant associations were determined by selecting the threshold-free cluster-enhancement option. To correct for multiple comparisons, a family-wise error-adjusted $P$ value $<.05$ was considered statistically significant because voxel-based analyses test thousands of voxels separately. If we controlled the family-wise error, the chance of $\geq 1$ false-positive across all voxels was lower than $5 \%$. Age, sex, height, brain volume, general physical performance, and variables that were univariatey associated with walking speed were entered as covariates.

ROI Analysis. To identify white matter tracts in which mean MTR correlates with gait velocity, we overlaid the probabilistic white matter tract atlas ${ }^{30}$ provided within FSL on significant voxels from the VMTRSM analysis.

The mean MTR within identified tracts was calculated in standard space by using FSLMATHS (http://fsl.fmrib.ox.ac.uk/fsl/ fslwiki/Fslutils). We eroded identified tracts by 1 voxel to reduce possible CSF contamination and partial volume effects from "edge" voxels. The mean MTR in the resulting "skeletonized" tracts was used in the subsequent linear multiple regression analysis.

A possible dose-effect relation of MTR within identified tracts and gait velocity was investigated by means of analysis of covariance with MTR in quartiles.

Age, sex, height, general physical performance, brain volume, and variables univariate-associated with walking speed were entered as covariates in the linear multiple regression analysis and the analysis of covariance. A $P$ value $<.05$ was considered statistically significant.

Voxel-Based Lesion Symptom Mapping. Nonparametric mapping was used to relate WMH location to gait velocity. ${ }^{37}$ One thousand permutations were performed to build the null distribution, and the Brunner-Munzel test was applied for statistical significance. ${ }^{38}$ Briefly, permutation testing is a procedure that compares a test statistic to a null distribution derived from the dataset of interest itself. Permuting how the dependent and independent variables are paired typically derives the permutation null distribution. When the null hypothesis is true (no effect), the observed pairings should be no more likely to generate an extreme test statistic than any other. ${ }^{39}$ Voxels affected in $<7$ subjects were not considered for analysis. Correction for multiple testing was 

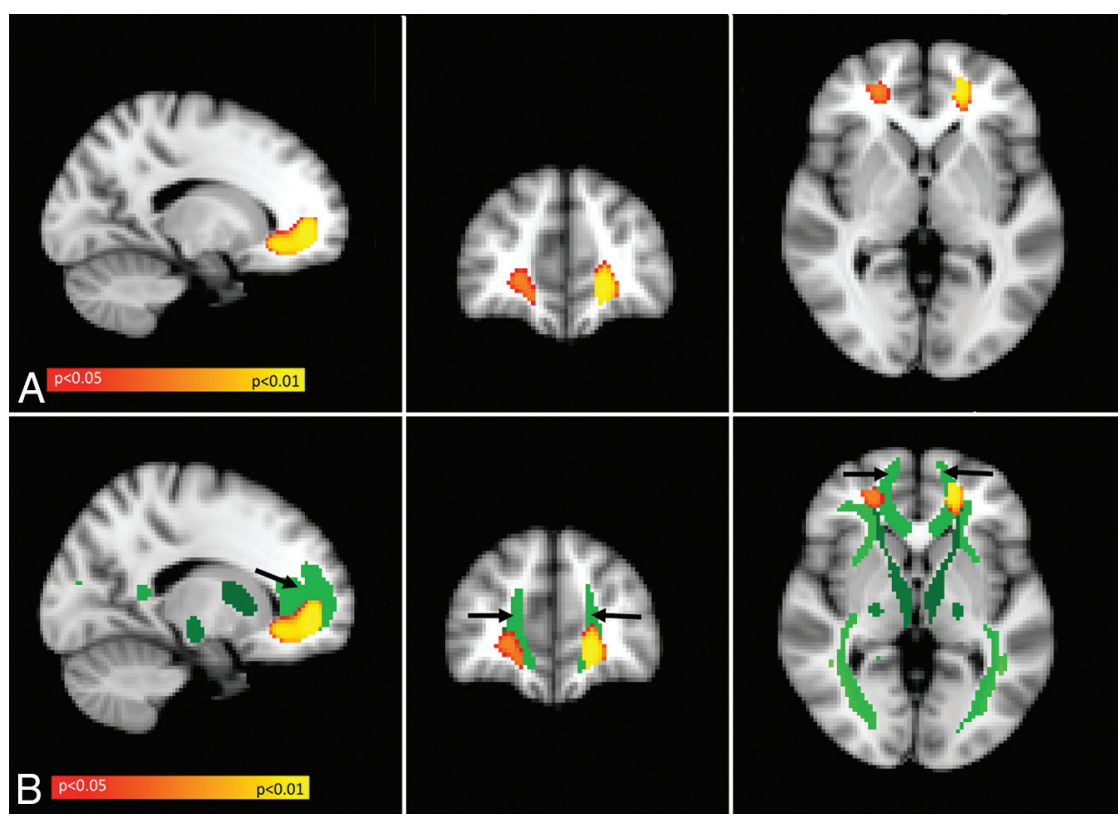

FIG 1. Result of voxel-based MTR symptom-mapping analysis. A, Yellow/orange represents MTR voxels positively related to gait velocity. The statistical map is superimposed on the MNI-152 standard space template and is family-wise error-corrected for multiple comparisons $(P<.05)$. The result is independent of age, sex, height, the presence of microbleeds, SPPB total score, diabetes, hyperuricemia, and brain volume. $B$, The statistical map shown in A (MTR voxels positively related to gait velocity) is now superimposed on the Johns Hopkins University DTI-based white matter tract atlas. Shades of green indicate different white matter tracts, as defined by the atlas. Most of the MTR voxels positively related to gait velocity (yellow/orange) are located within the forceps minor (light green frontal tract, indicated by black arrows).

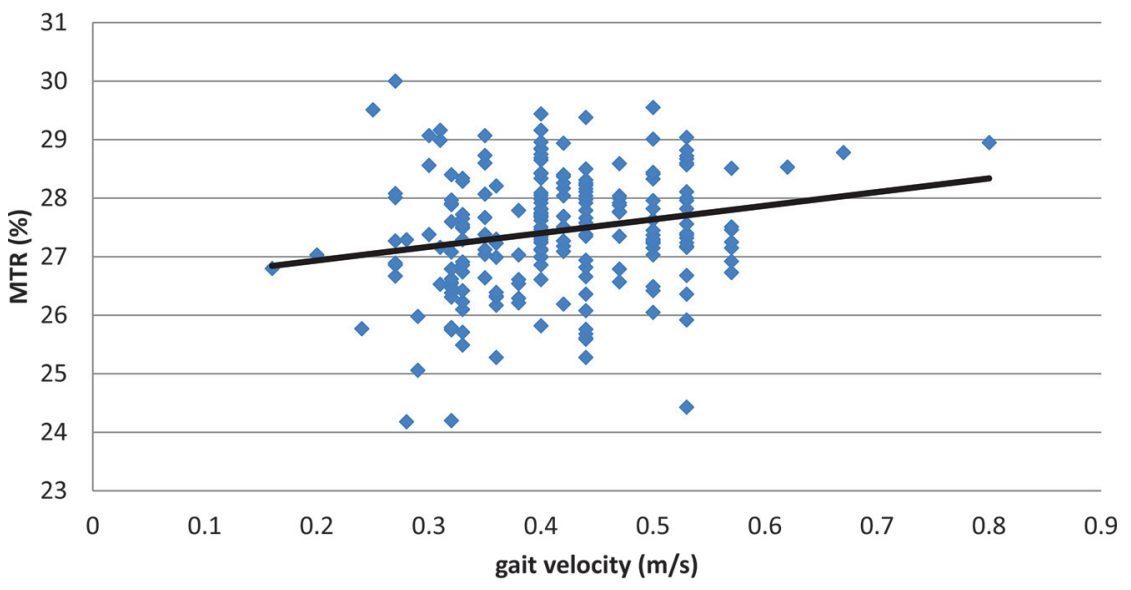

FIG 2. Correlation $(r=0.20 ; 95 \% \mathrm{Cl}, 0.08-0.32 ; P=.002$ ) between walking speed (x-axis, meters) second) and MTR within the forceps minor ( $y$-axis, percentage). $r$ indicates the Pearson correlation coefficient.

achieved by permutation-generated family-wise error thresholds. Age, sex, height, and brain volume were entered as covariates. To identify the localization of significant voxels within major white matter tracts, we used the probabilistic white matter tract atlas ${ }^{30}$ provided within FSL.

\section{RESULTS}

\section{Characteristics of the Study Population}

Demographic, clinical, and imaging characteristics of the study participants are summarized in the On-line Table. Individuals who walked slower were significantly older and shorter, their general physical performance was worse, and they more often had diabetes compared with their faster counterparts. Slower participants performed worse on executive function tasks and had more microbleeds on brain imaging. WMHs and cardiac disease did not relate to gait velocity.

\section{Imaging Data}

In the voxel-based MTR symptommapping analysis, we found significant clusters of MTR voxels that were positively correlated with gait velocity bilaterally within the frontal white matter (Fig 1A). The association remained significant after correction for multiple comparisons and adjustment for age, sex, height, brain volume, general physical performance, microbleeds, diabetes, and hyperuricemia. To examine the spatial relationship between these clusters and major white matter tracts, we projected significant clusters from the VMTRSM analysis on the probabilistic white matter tract atlas in MNI space. As shown in Fig $1 B$, there was substantial overlap with the forceps minor.

Given the prominent association of MTR voxels and walking speed within the forceps minor, we assessed the mean MTR of the forceps minor in standard space and used a linear regression model, adjusted for age, sex, height, brain volume, general physical performance, microbleeds, diabetes, and hyperuricemia to determine the association between forceps minor MTR and gait velocity. Higher mean MTR within the forceps minor was positively related to gait velocity ( $\beta=0.134 ; 95 \% \mathrm{CI}$, $0.011-0.258$; $P=.033$ ) (Fig 2). This association remained virtually unchanged when global WMH volume or WMH volume within the forceps minor was added to the analysis $(\beta=0.162 ; 95 \%$ CI, $0.024-0.307 ; P=.029$; and $\beta=$ 0.136; 95\% CI, 0.013-0.261; $P=.030$, respectively). Because the correlation between MTR and walking speed seemed to be dependent on a few outliers who walked quite fast, we repeated the regression analysis and excluded participants with walking speeds \pm 1.5 SDs from the population mean. This step did not substantially alter the direction or strength of the association ( $\beta=0.117 ; 95 \% \mathrm{CI},-0.012-0.248$; $P=.076)$.

We examined executive function as a confounder because it related significantly to gait velocity in the univariate analysis. Indeed, executive function attenuated the effect of forceps minor MTR on gait speed, and there remained only a nonsignificant trend $(\beta=$ 


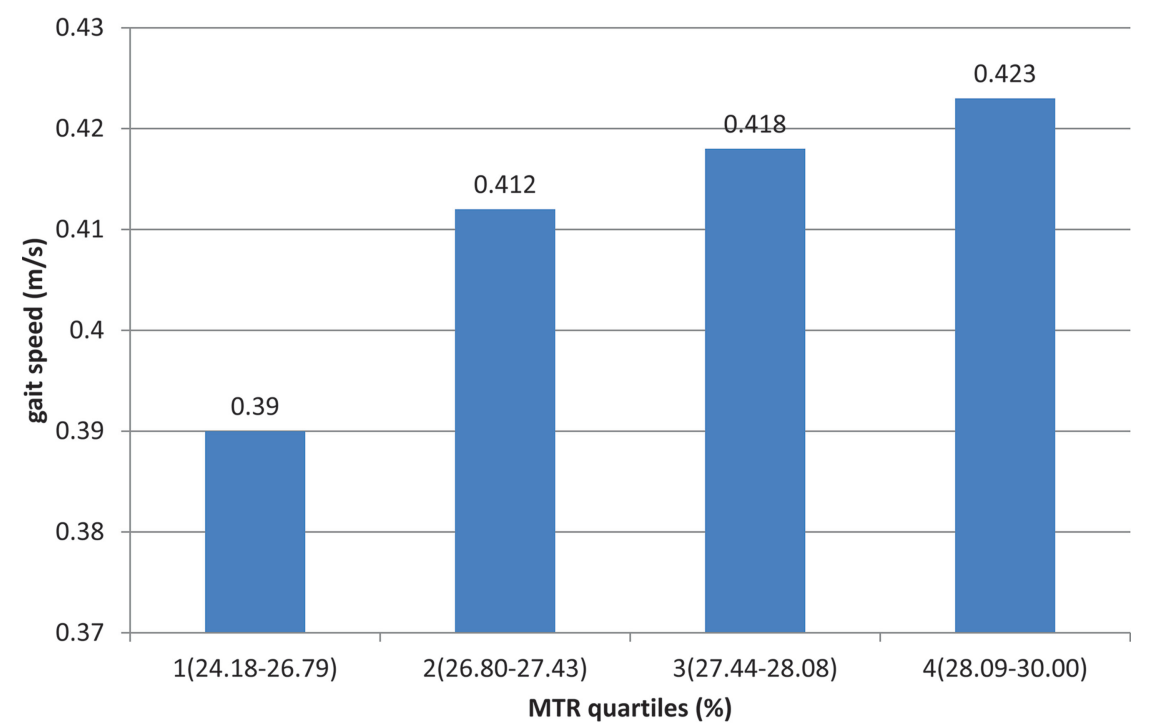

FIG 3. Analysis of covariance results. The mean MTR (percentage) of the forceps minor was divided into quartiles (x-axis). The first quartile was the lowest. Ranges of the MTR quartiles are as follows: quartile 1, 24.18-26.79; quartile 2, 26.80-27.43; quartile 3, 27.44-28.08; quartile 4, 28.0930.00. Values on the $y$-axis represent the estimated mean walking speed in meters/second of subjects within each quartile, adjusted for age, sex, height, SPPB total score, the presence of microbleeds, diabetes, hyperuricemia, and brain volume. Increasing MTR values within the forceps minor are related to higher gait velocity in a dose-dependent manner ( $P$ for linear trend $=.031$ ). only MTR study in healthy, older adults described a relationship between wholebrain MTR and gait velocity. ${ }^{23}$ The authors of this investigation quantified MTR changes globally by using histogram-based metrics. This is a very robust approach, but it fails to provide information on the location of MTR changes that relate to gait. Our voxelwise approach overcomes this limitation and thus extended previous results by identifying MTR alterations within the forceps minor as important determinants of walking speed. de Laat et $\mathrm{al}^{24}$ studied 429 individuals $50-85$ years of age with cerebral small-vessel disease. These authors found loss of white matter integrity in the corpus callosum, particularly the genu where the forceps minor crosses. Similar results were reported by Della Nave et $\mathrm{al}^{14}$ and Bhadelia et al, ${ }^{19}$ who also showed that participants with abnormal gait had lower fractional anisotropy in the genu of the 0.123; 95\% CI, $-0.002-0.243 ; P=.054)$. A Preacher and Hayes ${ }^{36}$ bootstrap method showed that executive dysfunction had no significant mediation effect on the association between MTR and gait velocity (indirect effect, 0.016; bootstrapped standard error, 0.013; bootstrapped 95\% CI, -0.0047-0.0460).

Figure 3 illustrates the associations between quartiles of mean MTR distribution in the forceps minor and gait velocity. Analysis of covariance, which we corrected for age, sex, height, brain volume, general physical performance, microbleeds, diabetes, and hyperuricemia, showed an independent linear dose-effect relationship between forceps minor-MTR quartiles and gait velocity $(P$ for linear trend $=.031)$.

VLSM identified no voxel clusters in which WMHs related significantly to slower gait speed.

\section{DISCUSSION}

In this study of older adults free of stroke, dementia, and other neurologic diseases, we used 2 observer-independent methods to identify micro- and macrostructural determinants of gait velocity. We found a dose-dependent association between MTR values in the forceps minor and gait velocity, independent of age, sex, height, general physical performance, diabetes, hyperuricemia, brain volume, microbleeds, and WMH volume. Lower MTR, suspected of representing decreased myelin content, ${ }^{17,22}$ related to slower gait velocity. VLSM showed no voxel clusters in which WMHs were significantly related to a slower gait velocity. Different results of MTR and WMH analysis in the current study are not surprising in light of the work of Wong et $\mathrm{al}^{40}{ }^{40}$ who reported no significant relationship among MTR, WMH, and cerebrovascular risk factors, suggesting an independent pathophysiology for each measure.

Our results are in line with MTR and DTI findings in normal aging and in patients with cerebral small-vessel disease. ${ }^{14,19,24}$ The corpus callosum. Even though DTI probes brain tissue organization rather than brain tissue composition like MTI, previous DTI results largely resemble our MTR findings. ${ }^{20,21}$ Microstructural brain tissue changes in the forceps minor were consistently linked with gait disturbances both in normal aging and in patients with cerebral small-vessel disease. ${ }^{14,19}$

The forceps minor is a large fiber bundle that connects the bilateral prefrontal cortices of the hemispheres, ${ }^{8,41}$ which play an important role in motor control, especially in older adults. ${ }^{42}$ Intact interhemispheric connections may be important for maintaining motor control at a high level. ${ }^{43}$ We observed no significant relationship between WMHs or lacunes and gait performance in our community-dwelling sample. Conversely, individuals with a higher number of microbleeds had slower gait velocity. This is partly in line with 1 recent study that reported an association between microbleeds, but not WMHs and gait velocity, in community-dwelling adults. ${ }^{44}$ Microbleeds occur in the wake of cerebral small-vessel disease, and their presence relates to microstructural brain tissue changes. ${ }^{45}$ However, neither the inclusion of WMHs nor of lacunes or microbleeds in the regression analysis changed the association between MTR of the forceps minor and gait velocity significantly.

The 2 previous DTI studies ${ }^{14,19}$ also found that DTI measures in the genu of the corpus callosum remained significantly associated with poorer gait performance after adding WMH volume to the analysis. Considering that more widespread WMHs seen in elderly individuals are a marker of coexisting cerebral small-vessel disease, previous results and the results of the current study indicate that factors other than cerebral small-vessel disease may also play an important role in the development of gait disturbances during aging. Postmortem studies that correlate MTR and DTI measures with brain tissue alterations are thus likely to im- 
prove our pathophysiologic understanding of age-related gait abnormalities.

The current study has several strengths. It is the largest cohort study on gait performance using voxel-based MTR mapping to date. The study is community-based, with prospectively planned radiologic and clinical protocols. With MTI, images with a much higher spatial resolution and signalto-noise ratio can be produced than is possible with DTI acquisitions. There are also no echo-planar imaging-induced artifacts. The high scan resolution allowed accurate registration of scans and segmentation of tissue types and WMHs. A thorough postprocessing procedure and rigorous quality control of segmentations reduced the effects of coregistration errors and CSF contamination to a minimum.

There are also limitations. The study has a cross-sectional design. Other potential underlying mechanisms for our findings, beyond a direct causal relationship between microstructural damage and disturbances in gait velocity, are possible. Among them are peripheral neuromuscular disease, the presence of hip and joint disease, or visual disturbances or cognitive dysfunction. We had expected peripheral neuromuscular disease and hip and joint diseases to be reflected in the SPPB total score, which was considered a confounder in our regression model. Inclusion of the SPPB total score did not significantly alter our results, arguing against a large effect of general physical performance on the relationship between forceps minor MTR and gait velocity.

When we added executive function scores to the regression analysis, the relationship between MTR of the forceps minor and walking speed was attenuated. There remained only a nonsignificant trend. In contrast to authors in previous studies who reported executive functions exerting mediating effects on the relationship between structural brain changes and gait, ${ }^{46,47}$ we were unable to confirm that executive function is a mediator in the association between forceps minor MTR and gait speed in the current investigation. It is likely that the effect of MTR on gait became nonsignificant when the executive function score was introduced simply because a trivial amount of variance was explained in addition to the model without inclusion of results of executive function testing. Participants were directed to walk at their "usual pace," allowing them to make a personal choice between a range of gait speeds. This could have biased our results. However, usual gait speed correlated well with the SPPB score in our study and was shown to relate significantly to disability in previous work. ${ }^{27}$ Previous data even suggest that usual gait speed alone is nearly as good a predictor of disability outcome as extensive physical testing. ${ }^{27}$

The lack of an association between heart disease and walking speed might have been caused by the rather short walking distance $(8 \mathrm{~m})$ and the possibility of walking at a normal pace in our study. It was described previously that the presence of heart disease rather affects long-distance walking tests. ${ }^{48}$ We realize that we tested the association between voxel values and walking speed in a far larger number of voxels in MTR analysis that compose the whole white matter than in the lesion-based analysis. Consequently, the risk of finding associations due to chance alone is probably higher in the MTR analyses, and by contrast, the risk of missing associations that actually exist is probably higher in the lesion-based analysis. In this study, besides family-wise error control, the symmetry of our findings in both hemispheres is a strong argument against findings due to chance alone.

The variance of the VMTRSM analyses was substantial. The reason for this large variability of results, which hampers the utility of findings, is unclear. It remains to be seen whether other methods of image analysis such as Tract-Based Spatial Statistics (TBSS; http://fsl.fmrib.ox.ac.uk/fsl/fslwiki/TBSS) ${ }^{49}$ might resolve this problem.

Some subjects originated from the same families. This might lead to correlated errors in the statistical models. FSL includes no regular option to correct voxelwise analyses for family structure. Including, for instance, random effects would be very difficult to interpret. However, we consider this a minor issue here, given the small number of subjects per family.

Our voxelwise approach is neither useful nor intended to diagnose a single subject. However, our results broaden the pathophysiologic understanding of MTR and gait disturbances in aging. Future longitudinal studies might use high-resolution, 3D MTR scans to tailor reliable predictors for gait disturbances in older age.

\section{CONCLUSIONS}

Our study provides new evidence for the importance of MTR changes in gait disturbances at an older age, particularly in the forceps minor. Identification of the causes and the histopathologic origin of these MR imaging-detected tissue alterations is important because therapeutic measures may be derived.

Disclosures: Stephan Seiler-RELATED: Grant: Comments: This work was supported by FP6 ERA-NET NEURON grant (01EW1207) and by the Austrian Science Fund project 1904*; Support for Travel to Meetings for the Study or Other Purposes: Comments: Travel costs for a study group meeting I attended (at Zuers, Austria) were covered by the grants indicated above.* Marco Duering—RELATED: Grant: European Union FP6 ERA-NET NEURON Grant*; Support for Travel to Meetings for the Study or Other Purposes: European Union FP6 ERA-NET NEURON Grant*; UNRELATED: Grants/ Grants Pending: German Center for Neurodegenerative Diseases Inter-Site Project Else Kröner-Fresenius-Stiftung*; Payment Lectures including Service on Speakers Bureaus: Bayer Vital GmbH, Comments: lectures. Jean-Francois Mangin—RELATED: Grant: Mechanisms of Small Vessel Related Brain Damage and Cognitive Impairment* Hugues Chabriat-UNRELATED: Board Membership: Servier, Comments: clinical trial preparation*; Consultancy: Johnson \& Johnson, Comments: Data and Safety Monitoring Board. Reinhold Schmidt-RELATED: Grant: FP6 ERA-NET NEURON grant (01EW1207) and the Austrian Science Fund project 1904.* *Money paid to the institution.

\section{REFERENCES}

1. Verghese J, LeValley A, Hall CB, et al. Epidemiology of gait disorders in community-residing older adults. J Am Geriatr Soc 2006;54: 255-61 CrossRef Medline

2. Mertz KJ, Lee DC, Sui X, et al. Falls among adults: the association of cardiorespiratory fitness and physical activity with walking-related falls. Am J Prev Med 2010;39:15-24 CrossRef Medline

3. Verghese J, Buschke H, Viola L, et al. Validity of divided attention tasks in predicting falls in older individuals: a preliminary study. J Am Geriatr Soc 2002;50:1572-76 CrossRef Medline

4. Kang HG, Dingwell JB. Effects of walking speed, strength and range of motion on gait stability in healthy older adults. J Biomech 2008; 41:2899-905 CrossRef Medline

5. Mahlknecht P, Kiechl S, Bloem BR, et al. Prevalence and burden of gait disorders in elderly men and women aged 60-97 years: a population-based study. PLoS One 2013;8:e69627 CrossRef Medline

6. la Fougère $C$, Zwergal $A$, Rominger $A$, et al. Real versus imagined locomotion: a [18F]-FDG PET-fMRI comparison. Neuroimage 2010;50:1589-98 CrossRef Medline 
7. Jones DK, Lythgoe D, Horsfield MA, et al. Characterization of white matter damage in ischemic leukoaraiosis with diffusion tensor MRI. Stroke 1999;30:393-97 CrossRef Medline

8. Catani M, ffytche DH. The rises and falls of disconnection syndromes. Brain 2005;128:2224-39 CrossRef Medline

9. Novak V, Haertle M, Zhao P, et al. White matter hyperintensities and dynamics of postural control. Magn Reson Imaging 2009;27: 752-59 CrossRef Medline

10. Murray ME, Senjem ML, Petersen RC, et al. Functional impact of white matter hyperintensities in cognitively normal elderly subjects. Arch Neurol 2010;67:1379-85 CrossRef Medline

11. Baezner H, Blahak C, Poggesi A, et al; LADIS Study Group. Association of gait and balance disorders with age-related white matter changes: the LADIS study. Neurology 2008;70:935-42 CrossRef Medline

12. Poggesi A, Gouw A, van der Flier W, et al. Cerebral white matter changes are associated with abnormalities on neurological examination in non-disabled elderly: the LADIS study. J Neurol 2013;260: 1014-21 CrossRef Medline

13. Soumare A, Elbaz A, Zhu Y, et al. White matter lesions volume and motor performances in the elderly. Ann Neurol 2009;65:706-15 CrossRef Medline

14. Della Nave R, Foresti S, Pratesi A, et al. Whole-brain histogram and voxel-based analyses of diffusion tensor imaging in patients with leukoaraiosis: correlation with motor and cognitive impairment. AJNR Am J Neuroradiol 2007;28:1313-19 CrossRef Medline

15. Guttmann CR, Benson R, Warfield SK, et al. White matter abnormalities in mobility-impaired older persons. Neurology 2000;54: 1277-83 CrossRef Medline

16. Seiler S, Pirpamer L, Hofer E, et al. Magnetization transfer ratio relates to cognitive impairment in normal elderly. Front Aging $\mathrm{Neu}$ rosci 2014;6:263 CrossRef Medline

17. Seiler S, Ropele S, Schmidt R. Magnetization transfer imaging for in vivo detection of microstructural tissue changes in aging and dementia: a short literature review. J Alzheimers Dis 2014;42(suppl 3):S229-37 CrossRef Medline

18. Tuladhar AM, van Norden AG, de Laat KF, et al. White matter integrity in small vessel disease is related to cognition. Neuroimage Clin 2015;7:518-24 CrossRef Medline

19. Bhadelia RA, Price LL, Tedesco KL, et al. Diffusion tensor imaging, white matter lesions, the corpus callosum, and gait in the elderly. Stroke 2009;40:3816-20 CrossRef Medline

20. Mori S, Zhang J. Principles of diffusion tensor imaging and its applications to basic neuroscience research. Neuron 2006;51:527-39 CrossRef Medline

21. Graham SJ, Henkelman RM. Understanding pulsed magnetization transfer. J Magn Reson Imaging 1997;7:903-12 CrossRef Medline

22. Schmierer K, Scaravilli F, Altmann DR, et al. Magnetization transfer ratio and myelin in postmortem multiple sclerosis brain. Ann Neurol 2004;56:407-15 CrossRef Medline

23. Rosano C, Sigurdsson S, Siggeirsdottir K, et al. Magnetization transfer imaging, white matter hyperintensities, brain atrophy and slower gait in older men and women. Neurobiol Aging 2010;31:1197204 CrossRef Medline

24. de Laat KF, Tuladhar AM, van Norden AG, et al. Loss of white matter integrity is associated with gait disorders in cerebral small vessel disease. Brain 2011;134:73-83 CrossRef Medline

25. Schmidt R, Lechner H, Fazekas F, et al. Assessment of cerebrovascular risk profiles in healthy persons: definition of research goals and the Austrian Stroke Prevention Study (ASPS). Neuroepidemiology 1994;13:308-13 CrossRef Medline

26. Schmidt R, Fazekas F, Kapeller P, et al. MRI white matter hyperintensities: three-year follow-up of the Austrian Stroke Prevention Study. Neurology 1999;53:132-39 CrossRef Medline

27. Guralnik JM, Ferrucci L, Pieper CF, et al. Lower extremity function and subsequent disability: consistency across studies, predictive models, and value of gait speed alone compared with the short physical performance battery. J Gerontol A Biol Sci Med Sci 2000;55: M221-31 CrossRef Medline
28. Greenberg SM, Vernooij MW, Cordonnier C, et al; Microbleed Study Group. Cerebral microbleeds: a guide to detection and interpretation. Lancet Neurol 2009;8:165-74 CrossRef Medline

29. Plummer DL. DispImage: a display and analysis tool for medical images. Revista di Neuroradiologica 1992;5:489-95 CrossRef

30. Hua K, Zhang J, Wakana S, et al. Tract probability maps in stereotaxic spaces: analyses of white matter anatomy and tract-specific quantification. Neuroimage 2008;39:336-47 CrossRef Medline

31. Jenkinson M, Beckmann CF, Behrens TE, et al. FSL. Neuroimage 2012;62:782-90 CrossRef Medline

32. Zhang Y, Brady M, Smith S. Segmentation of brain MR images through a hidden Markov random field model and the expectationmaximization algorithm. IEEE Trans Med Imaging 2001;20:45-57 CrossRef Medline

33. Smith SM. Fast robust automated brain extraction. Hum Brain Mapp 2002;17:143-55 CrossRef Medline

34. Jenkinson M, Smith S. A global optimisation method for robust affine registration of brain images. Med Image Anal 2001;5:143-56 CrossRef Medline

35. Ashburner J, Friston KJ. Voxel-based morphometry: the methods. Neuroimage 2000;11:805-21 CrossRef Medline

36. Preacher KJ, Hayes AF. Asymptotic and resampling strategies for assessing and comparing indirect effects in multiple mediator models. Behav Res Methods 2008;40:879-91 CrossRef Medline

37. Rorden C, Karnath HO, Bonilha L. Improving lesion-symptom mapping. J Cogn Neurosci 2007;19:1081-88 CrossRef Medline

38. Kimberg DY, Coslett HB, Schwartz MF. Power in voxel-based lesionsymptom mapping. J Cogn Neurosci 2007;19:1067-80 CrossRef Medline

39. Winkler AM, Ridgway GR, Webster MA, et al. Permutation inference for the general linear model. Neuroimage 2014;92:381-97 CrossRef Medline

40. Wong KT, Grossman RI, Boorstein JM, et al. Magnetization transfer imaging of periventricular hyperintense white matter in the elderly. AJNR Am J Neuroradiol 1995;16:253-38 Medline

41. Montero-Odasso M, Verghese J, Beauchet O, et al. Gait and cognition: a complementary approach to understanding brain function and the risk of falling. J Am Geriatr Soc 2012;60:2127-36 CrossRef Medline

42. Chao YP, Cho KH, Yeh CH, et al. Probabilistic topography of human corpus callosum using cytoarchitectural parcellation and high angular resolution diffusion imaging tractography. Hum Brain Mapp 2009;30:3172-87 CrossRef Medline

43. Seidler RD, Bernard JA, Burutolu TB, et al. Motor control and aging: links to age-related brain structural, functional, and biochemical effects. Neurosci Biobehav Rev 2010;34:721-33 CrossRef Medline

44. Stijntjes M, de Craen AJ, van der Grond J, et al. Cerebral microbleeds and lacunar infarcts are associated with walking speed independent of cognitive performance in middle-aged to older adults. Gerontology 2016;62:500-07 CrossRef Medline

45. Akoudad S, de Groot M, Koudstaal PJ, et al. Cerebral microbleeds are related to loss of white matter structural integrity. Neurology 2013;81:1930-37 CrossRef Medline

46. Ezzati A, Katz MJ, Lipton ML, et al. The association of brain structure with gait velocity in older adults: a quantitative volumetric analysis of brain MRI. Neuroradiology 2015;57:851-61 CrossRef Medline

47. Nadkarni NK, Nunley KA, Aizenstein H, et al; Health ABC Study. Association between cerebellar gray matter volumes, gait speed, and information-processing ability in older adults enrolled in the health $\mathrm{ABC}$ study. J Gerontol A Biol Sci Med Sci 2014;69:996-1003 CrossRef Medline

48. Pasma JH, Stijntjes M, Ou SS, et al. Walking speed in elderly outpatients depends on the assessment method. Age (Dordr) 2014;36:9736 CrossRef Medline

49. Smith SM, Jenkinson M, Johansen-Berg H, et al. Tract-based spatial statistics: voxelwise analysis of multi-subject diffusion data. Neuroimage 2006;31:1487-505 CrossRef Medline 